\title{
A Case Report of Reversal with Sugammadx in a Myasthenic Patient
}

\author{
Ju Han Mun ${ }^{1}$, Cheol Jong Woo ${ }^{1}$, Jeongwoo Lee ${ }^{1}$, Dong-Chan Kim ${ }^{1}$, Jun Ho Lee ${ }^{1 *}$ \\ ${ }^{1}$ Department of Anesthesiology and Pain Medicine, Jeonbuk National University Medical \\ School and hospital, Jeonju, Korea
}

\section{Abstract}

Background/Objectives: Myasthenia gravis (MG) is an autoimmune disease caused by the development of antibodies against the nicotinic acetylcholine receptor. Methods/Statistical analysis: MG causes weakness and fatigue of the respiratory muscles, so that there is hypersensitivity against non-depolarizing muscle relaxants. In general acetylcholinesterase inhibitors are administered to reverse the muscle blockade, but the use of neuromuscular blocking agents in myasthenic patients is usually delayed due to the drugs that already inhibit cholinesterase, resulting in a delay in spontaneous respiration. Sugammadex eliminates the effects of steroid non-depolarizing muscle relaxants, such as rocuronium and vecuronium, by selectively encapsulating their molecules. Case: We present a case of reversal of neuromuscular blocking agent and return to spontaneous respiration with sugammadex in a myasthenic patient. Findings: We also evaluated the degree of spontaneous respiration by comparing pulmonary function tests before and after surgery in a MG patient. Improvements/Applications: Sugammadex is a good option of fast reversal of neuromuscular blocking agent and return to spontaneous respiration in a myasthenic patient.

\section{Index Terms}

Myasthenia gravis, Sugammadex, Nicotinic acetylcholine receptor, Acetylcholinesterase inhibitors, Cholinesterase, Neuromuscular blocking

\section{Corresponding author: Jun Ho Lee}

gojuno@jbnu.ac.kr

- Manuscript received July 1, 2020.

- Revised August 10,2020; Accepted September 2, 2020

- Date of publication September 30, 2020.

(c) The Academic Society of Convergence Science Inc.

2619-8363 @ 2020 IJBSA. Personal use is permitted, but republication/redistribution requires IJBSA permission. 


\section{INTRODUCTION}

Myasthenia gravis (MG) is an
autoimmune disease caused by the
development of antibodies against the
nicotinic acetylcholine receptor [1]. The
symptoms of MG are muscle fatigue and
variable weakness. The muscular weakness
may cause aspiration pneumonia, dysarthria,
or even swallowing difficulty. During
anesthesia, neuromuscular blocking
agents(NMBAs) are administered for
immobile surgical field [2]. NMBAs blocks
targets the neuromuscular junction, and
offers skeletal muscle relaxation for the
operation. They also lead to respiratory
arrest as well. This effect is reversed with
acetylcholinesterase inhibitors. The increase
of acetylcholine by the acetylcholinesterase
inhibitors promotes a rapid return of
spontaneous respiration.

In normal condition the adequate dosage of NMBAs are administered, but the dose cannot be determined optimally in certain condition such as MG. If there is a thymoma present, myasthenic symptom can be presented. Thymoma is most often a benign tumor of the thymus which produces acetylcholine receptor autoantibodies and can sometimes even produce autoantibodies. Those patients usually take anticholinesterase inhibitor for symptom relieving effect. Thus, the recovery from NMBAs through the administration of further acetylcholinesterase inhibitors might be delayed after surgery. It may lead the residual neuromuscular blockade and postoperative respiratory failure. Also, insufficient muscle strength may result in hypoventilation, oxygen desaturation, development of hypercapnia, aspirations, and postoperative bronchopneumonia in the postoperative period [3].

Sugammadex is a rapid steroidal reversal agent from the neuromuscular blocking effect from NMBAs. Unlike the mechanism of acetylcholinesterase inhibitors, sugammadex binds directly to NMBAs, quickly reversing the neuromuscular blocking effect of the NMBAs and facilitating spontaneous respiration [4]. Efficiency and reliability of sugammadex has been repeatedly proven in clinical field. Here, we report a case of MG patient who received general anesthesia for spinal surgery. The patient has recovered from the effects of NMBA rapidly by using sugammadex. We also evaluated the degree of spontaneous respiration by comparing pulmonary function tests before and after surgery in the MG patient.

\section{CASE REPORT}

A 58-year-old female was admitted for scheduled posterolateral internal fixation due to Lumbar spinal stenosis L3-S1. She had been diagnosed with MG and had undergone thymectomy 10 years ago, but the symptoms such as diplopia, dysphagia and difficulty to lift heavy things were returned after thymectomy. Also, she had DM and hypertension. At the time of operation, medications included prednisolone, pyridostigmin $60 \mathrm{mg}$, azathioprine, ACE inhibitor and metformin. Detailed explanation of the respiratory risks of MG undergoing general anesthesia and information about sugammadex was offered with informed consent.

Preoperative laboratory tests were within normal range including hemoglobin 11.9 $\mathrm{mg} / \mathrm{dl}$ and Prothrombin Time International Normalized Ratio (INR) 1.01. Also, electrolyte, liver function test, chest PA, electrocardiography (ECG) and echochardiogram presented non-specific. Preoperative pulmonary function test (PFT) presented FEV1 $1.78 \mathrm{~L}(99 \%)$ and FVC $1.87 \mathrm{~L}(89 \%)$.

After arriving at the operating room, ECG, blood pressure, pulse oximetry and a 
peripheral nerve stimulator (TOF watch $\AA$, Organon Ltd., Dublin, Ireland) were monitored. Initial vital sign was blood pressure 130/64 $\mathrm{mmHg}$, heart rate $65 /$ minute, respiratory rate $20 /$ minute, Oxygen saturation $94 \%$ After preoxygenation for 3 minutes with $100 \%$ $\mathrm{O} 2$, anesthesia was induced by $1 \%$ propofol $100 \mathrm{mg}$ and remifentanil (Ultiva $\AA$, GlaxoSmithKline, Parma, Italy). Remifentanil was infused using effect-site target-controlled infusion pump (Orchestra ${ }^{\circledR}$ Base Primea, Fresenius Vial, Brézins, France) with Minto model. Initially, patients were induced with remifentanil 4 $\mathrm{ng} / \mathrm{ml}$ of the effect-site concentration and 3 vol $\%$ of sevoflurane with $\mathrm{FiO} 20.5(\mathrm{O} 2+$ Air). After patients lost consciousness, 1.0 $\mathrm{mg} / \mathrm{kg}$ of rocuronium were administered. The patient were manually ventilated via a face mask with air in oxygen, and the anesthesiologist tried to keep fixed tidal volume $8-10 \mathrm{ml} / \mathrm{kg}$ under spirometric monitoring. Orotracheal intubation was performed at 3 min after loss of consciousness when train-of-four count was 0 (4 stimuli at $2 \mathrm{~Hz}, 50 \mathrm{~mA}$ ), and effect-site concentration of remifentanil was steadystate levels at 3-3.5 ng/ml. Intubation was done with Armored tube \#7.0, and fixed at $21 \mathrm{~cm}$ from the incisor. After endotracheal intubation, controlled mechanical ventilation was maintained with a TV 8 $\mathrm{ml} / \mathrm{kg}$ (based on ideal body weight) and an inspiratory to expiratory ratio 1:2 with $5 \mathrm{cmH}_{2} \mathrm{O}$ PEEP. The ventilatory frequency was set to maintain an end-tidal $\mathrm{PCO}_{2}$ range of $38-42 \mathrm{mmHg}$. After induction esophageal temperature was monitored. Arterial line was secured for continuous BP monitoring and cardiac index. Central line was secured with $8 \mathrm{Fr}$. double lumen at the right subclavian vein and central venous pressure was monitored through out the operation. The patient was actively warmed to keep the body temperature $36.0-36.5^{\circ} \mathrm{C}$ by using a forced air warming unit and a fluid line warmer. The patient's muscular relaxation, blood pressure, heart rate, peripheral oxygen saturation, and temperature were recorded throughout the operation (Table 1), (Table 2).

Table 1. Record throughout the operation 1.

\begin{tabular}{|l|l|l|l|l|}
\hline & LOC & Rocuronium & Intubation & End of OP \\
\hline Time & $58 \mathrm{~s}$ & $\begin{array}{l}114 \mathrm{~s} \\
\left(1^{\prime} 54^{\prime},\right)\end{array}$ & $\begin{array}{l}270 \mathrm{~s} \\
\left(4^{\prime} 30^{\prime \prime}\right)\end{array}$ & $4 \mathrm{~h} 15 \mathrm{~min}$ \\
\hline TOF & $100 \%$ & $80 \%$ & 0 & $68 \%$ \\
\hline PR & 58 & 56 & 62 & 76 \\
\hline BP & $129 / 57$ & $95 / 58$ & $83 / 49$ & $129 / 58$ \\
\hline Temp & - & - & - & 35.8 \\
\hline BIS & 51 & 45 & 46 & 62 \\
\hline
\end{tabular}

Table 2. Record throughout the operation 2.

\begin{tabular}{|l|l|l|l|}
\hline & $\begin{array}{l}\text { Sugammadex } \\
\text { injection }\end{array}$ & TOF ratio 0.9 & Obey \\
\hline Time & $\begin{array}{l}180 \mathrm{~s} \\
\left(3^{\prime} 0^{\prime \prime}\right)\end{array}$ & $\begin{array}{l}109 \mathrm{~s} \\
\left(19^{\prime}, 9^{\prime}\right)\end{array}$ & $\begin{array}{l}320 \mathrm{~s} \\
\left(6^{\prime} 20^{\prime \prime}\right)\end{array}$ \\
\hline TOF & $76 \%$ & $90 \%$ & $100 \%$ \\
\hline PR & 74 & 92 & 94 \\
\hline BP & $119 / 57$ & $141 / 63$ & $165 / 85$ \\
\hline Temp & 35.8 & 35.8 & - \\
\hline BIS & 69 & 82 & 92 \\
\hline
\end{tabular}

During the operation, general anesthesia was maintained with sevoflurane 0.8-1.0 
Mac (1.7-2.0 vol\%) and remifentanil $0.01-0.20 \mu \mathrm{g} / \mathrm{kg} / \mathrm{min}$ according to the values of BIS 40-60. The duration of the operation was 4 hours and 15 minutes. The amount of fluid was about $3900 \mathrm{ml}$ crystalloid and 500 $\mathrm{ml}$ colloid during the period of the operation. Three packs of packed RBC was transfused. Pain control was done by Patient Controlled Analgesia with a regimen of fentanyl 700 $\mathrm{mg}$, ketorolac $150 \mathrm{mg}$ and nefopam 100mg in volume of $60 \mathrm{~mL}$ mixture. The operation was done without any complication.

After the operation ended, sugammadex $120 \mathrm{mg}(2 \mathrm{mg} / \mathrm{kg})$ was administered to recovery spontaneous respiration. When the value of the TOF increased to 0.9 (109 seconds after the sugammadex injection), the patient was extubated. Three hundred and twenty seconds after sugammadex injection, verbal command was obeyed. Then, the patient was transferred to postanesthesia care unit.

The patient's blood pressure, peripheral oxygen saturation, and heart rate were observed for 30 minutes postoperatively, and no specific symptoms were observed in the post-anesthesia care unit. Preoperative pulmonary function test (PFT) presented FEV1 1.91 L (89\%) and FVC 1.82 L (99\%). The patient was discharged without an event.

\section{Discussion}

Myasthenia gravis patients, particularly those undergoing major surgery and/or suffering from concomitant disorders, require special individual management in preparation for surgery, appropriate selection and administration of anesthesia, and close monitoring postoperatively $[5,6]$.

Sugammadex is an NMBA antagonist and has a different mechanism from the usual acetylcholinesterase inhibitors. It binds directly to the NMBAs and reverses their neuromuscular blocking effects. According to Jones et al., when comparing sugammadex and a combination of glycopyrrolate and neostigmine under general anesthesia with rocuronium 0.6 $\mathrm{mg} / \mathrm{kg}$, the group that used sugammadex recovered significantly faster [7].

Sugammadex allows for faster recovery than conventional reversal agents. It is therefore a particularly useful alternative for patients with MG. Rudzka-Nowak and Piechota reported that a patient with myasthenia gravis who had undergone abdominal surgery and was administered 3 $\mathrm{mg} / \mathrm{kg}$ of sugammadex intravenously completely recovered spontaneous respiration within 5 minutes [8].

All the beneficial qualities of sugammadex offer the possibility for using the drug in patients who are expected to have problems with proper ventilation and gas exchange. In the case described here, sugammadex made it possible to perform a safe general anaesthesia procedure with skeletal muscle relaxants without prolonging mechanical ventilation. The medical literature contains isolated reports on the use of sugammadex (with success) in patients with MG. We performed pulmonary function test to compare the ability of spontaneous respiration. PFT is a complete evaluation of the respiratory system so that it identifies the severity of pulmonary impairment after general anesthesia. In this case, preoperative and postoperative PFT were unchanged, which means the patient with $\mathrm{MG}$ was reversed completely after use of NMBAs.

The authors believe that sugammadex as an agent used for muscle relaxation in delivering anesthesia to MG patients may be the gold standard. Sugammadex, used in combination with objective neuromuscular monitoring, can be applied to reverse rocuronium induced neuromuscular blockade in patients with MG. 


\section{REFERENCES}

[1] Özel, F., Altunkan, A. A., \& Azizoğlu, M. (2016). Postoperative Respiratory Failure in a Patient with Undiagnosed Myastenia Gravis. Turkish journal of anaesthesiology and reanimation, 44, 108-110.

DOI: 10.5152/TJAR.2016.03274.

[2] Kim, R. K., \& Kim, S. Y. (2016). Rapid Return of Spontaneous Respiration after General Anesthesia with Sugammadex in a Patient with Myasthenia Gravis. Journal of lifestyle medicine, 6(1), 43-46.

DOI: $10.15280 / \mathrm{j} \mathrm{lm} .2016 .6 .1 .43$

[3] Vymazal, T., Krecmerova, M., Bicek, V., \& Lischke, R. (2015). Feasibility of full and rapid neuromuscular blockade recovery with sugammadex in myasthenia gravis patients undergoing surgery - a series of 117 cases. Therapeutics and clinical risk management, 11, 1593-1596. DOI: 10.2147/TCRM.S93009.

[4] Rex, C., \& Pühringer, F. K. (2009). Sugammadex-a new era in the antagonsim of muscle relaxants. Anasthesiologie, Intensivmedizin, Notfallmedizin, Schmerztherapie: AINS 44(5), 368-374; quiz 76, 79 DOI: $10.1055 / \mathrm{s}-0029-1224784$

[5] Dillon, F. X. (2004). Anesthesia issues in the perioperative management of myasthenia gravis. Seminars in neurology, 24(1), 83-94.

DOI: $10.1055 / \mathrm{s}-2004-829587$

[6] Unterbuchner, C., Fink, H., \& Blobner, M. (2010). The use of sugammadex in a patient with myasthenia gravis. Anaesthesia, 65(3), 302-305. DOI: $10.1111 / \mathrm{j} .1365-2044.2009 .06236 . x$.

[7] Jones, R. K., Caldwell, J. E., Brull, S. J., \& Soto, R. G. (2008). Reversal of profound rocuronium-induced blockade with sugammadex: a randomized comparison with neostigmine. Anesthesiology, 109(5), 816-824.

DOI: $10.1097 / A L N .0 b 013 \mathrm{e} 31818 \mathrm{a}$ fee. 